\title{
CONSTRUCTION OF COSPECTRAL INTEGRAL REGULAR GRAPHS
}

\author{
RAVINDRA B. BAPAT ${ }^{1}$ \\ Indian Statistical Institute \\ Delhi Centre, 7 S.J.S.S. Marg \\ New Delhi 110 016, India \\ e-mail: rbb@isid.ac.in \\ AND \\ MASOUD KARIMI $^{2}$ \\ School of Mathematical Sciences \\ Anhui University, Hefei, China \\ and \\ Department of Mathematics, Bojnourd Branch \\ Islamic Azad University, Bojnourd, Iran \\ P.O. Box 94176-94686 \\ e-mail: karimimth@yahoo.com
}

\begin{abstract}
Graphs $G$ and $H$ are called cospectral if they have the same characteristic polynomial. If eigenvalues are integral, then corresponding graphs are called integral graph. In this article we introduce a construction to produce pairs of cospectral integral regular graphs. Generalizing the construction of $G_{4}(a, b)$ and $G_{5}(a, b)$ due to Wang and Sun, we define graphs $\mathcal{G}_{4}(G, H)$ and $\mathcal{G}_{5}(G, H)$ and show that they are cospectral integral regular when $G$ is an integral $q$ regular graph of order $m$ and $H$ is an integral $q$-regular graph of order $(b-2) m$ for some integer $b \geq 3$.
\end{abstract}

Keywords: eigenvalue, cospectral graphs, adjacency matrix, integral graphs.

2010 Mathematics Subject Classification: 05C50.

\section{REFERENCES}

\footnotetext{
${ }^{1}$ This author acknowledges support from JC Bose Fellowship awarded by the Department of Science and Technology, Government of India.

${ }^{2}$ Corresponding author.
} 
[1] K.T. Balińska, M. Kupczyk, S.K. Simić and K.T. Zwierzyński, On generating all integral graphs on 11 vertices, Computer Science Center Report No. 469, Technical University of Poznań (1999/2000) 1-53.

[2] K.T. Balińska, M. Kupczyk, S.K. Simić and K.T. Zwierzyński, On generating all integral graphs on 12 vertices, Computer Science Center Report No. 482, Technical University of Poznań (2001) 1-36.

[3] R.B. Bapat, Graphs and Matrices (Springer, 2014). doi:10.1007/978-1-4471-6569-9

[4] A.E. Brouwer and W. Haemers, Spectra of Graphs (Springer, 2012). doi:10.1007/978-1-4614-1939-6

[5] F.C. Bussemaker and D.M. Cvetković, There are exactly 13 connected, cubic, integral graphs, Univ. Beograd. Publ. Elektrothen 552 (1976) 43-48.

[6] D.M. Cvetković, M. Doob and H. Sachs, Spectra of Graphs (Academic Press, New York, 1980).

[7] C.D. Godsil and B.D. McKay, Constructing cospectral graphs, Aequationes Math. 25 (1982) 257-268 doi:10.1007/BF02189621

[8] A.J. Schwenk, Exactly thirteen connected cubic graphs have integral spectra, in: Theory and Applications of Graphs, Y. Alavi and D.R. Lick (Ed(s)), (Springer, 1978) Lecture Notes in Math. 642 516-533. doi: $10.1007 / \mathrm{bfb} 0070407$

[9] E.R. van Dam, Graphs with few eigenvalues; an interplay between combinatorics and algebra (Ph.D. Thesis, Tilburg University, Center for Economic Research Dissertation Series, No. 20, 1996).

[10] L. Wang and H. Sun, Infinitely many pairs of cospectral integral regular graphs, Appl. Math. J. Chinese Univ. 26 (2011) 280-286. doi:10.1007/s11766-011-2180-1

[11] L. Wang, X. Li and C. Hoede, Two classes of integral regular graphs, Ars Combin. 76 (2005) 303-319.

[12] D.B. West, Introduction to Graph Theory (Prentice Hall, Inc., Upper Saddle River, NJ, 1996).

Received 28 December 2015

Revised 27 May 2016

Accepted 27 May 2016 